\title{
Method for Simulating the Performance of a Boundary Layer Ingesting Propulsion System at Design and Off-design
}

\author{
C. Goldberg, D. Nalianda, D. MacManus, and P. Pilidis \\ Cranfield University, Bedfordshire, United Kingdom \\ J. Felder
}

NASA Glenn Research Center, Cleveland, Ohio, USA

\begin{abstract}
Boundary layer ingestion has emerged as a potential propulsion concept on novel aircraft configurations for the future. As these concepts progress, preliminary design tools are required that enable the simulation of these aircraft and the rapid analysis of multiple configurations. Simulation tools for boundary layer ingesting propulsion systems tend to focus on proving performance benefits at design point. However, the simulation of aircraft configurations that utilise boundary layer ingestion requires a method to simulate the propulsion system at a range of flight conditions other than design point. A tool is therefore required to enable simulations at off-design. This research presents a work flow to simulate a boundary layer ingesting propulsion system at design and off-design. The process is intended as a tool for design space exploration and the rapid analysis of concepts at the conceptualisation phase. Boundary layer calculations have been combined with conventional 1-D gas turbine performance methods to predict performance of a propulsion system at design point. This method is then extended to enable simulations at off-design conditions for a range of flight conditions or propulsion system power settings. The formulation provides a thrust-drag representation that supports conventional aircraft simulation tools. A case study of an aircraft configuration which utilises an array of boundary layer ingesting propulsors is used to demonstrate the process. The performance of individual propulsors in the array is compared at off-design. Simulations found that, although each propulsor was sized for the same propulsive force at design point, off-design performance diverged depending on operating conditions. In addition, the performance of the propulsor array as a whole was predicted as a function of altitude and Mach number. The case study is used to draw general conclusions on the performance characteristics of a boundary layer ingesting propulsor.
\end{abstract}

Keywords: Propulsion modelling, Aircraft propulsion, Novel propulsion systems, Boundary layer ingestion

\section{Nomenclature}

$\delta \quad$ Boundary layer thickness (m)

$\delta^{*} \quad$ Displacement thickness (m)

$\dot{m} \quad$ Mass flow rate $(\mathrm{kg} / \mathrm{s})$

$\rho \quad$ Density $\left(\mathrm{kg} / \mathrm{m}^{3}\right)$

$\tau_{w} \quad$ Shear stress $\left(\mathrm{N} / \mathrm{m}^{2}\right)$

A Area $\left(\mathrm{m}^{2}\right)$

c $\quad$ Aircraft chord length (m)

$D \quad \operatorname{Drag}(\mathrm{N})$

$F_{G} \quad$ Gross thrust (N)

$F_{N} \quad$ Net thrust (N)

$h \quad$ Streamtube height (m)
$P$
Total pressure $(\mathrm{Pa})$
$p \quad$ Static pressure $(\mathrm{Pa})$
$P_{B L I} \quad$ BLI propulsion system power (MW)
$P_{\text {ref }} \quad$ Reference propulsion system power (MW)
$S_{\text {wet }} \quad$ Wetted surface area $\left(\mathrm{m}^{2}\right)$
$u \quad$ Axial velocity $(\mathrm{m} / \mathrm{s})$
$w \quad$ Streamtube width (m)
$x \quad$ Chordwise distance from leading edge $(\mathrm{m})$
$x_{0} \quad$ Chordwise distance from aircraft nose (m)
$y \quad$ Vertical distance above surface (m)
$z \quad$ Spanwise distance from centreline (m)
BLI Boundary layer ingestion 
FPR Fan pressure ratio

FS Free-stream

MAG Mass flow non-dimensional group

NPF Net propulsive force

PSC Power saving coefficient

Re Reynolds Number

SPC Specific power consumption

\section{Introduction}

Aircraft concepts are being developed that make use of novel configurations to achieve improvements in efficiency through performance benefits to the airframe and propulsion system. Boundary layer ingestion (BLI) is one such technology that has been implemented in a number of conceptual designs. In an aircraft, the boundary layer contributes to drag and leads to a momentum deficit, or wake. The boundary layer can be similarly detrimental to the propulsion system, as a turbulent boundary layer gives rise to non-uniformities in the flow which negatively impact performance. Conventional propulsion system design therefore typically seeks to avoid ingesting any boundary layer flow. However, boundary layer ingestion provides a way in which the boundary layer may be used to improve the overall efficiency of the aircraft and reduce fuel consumption. The resulting lower momentum of the boundary layer flow reduces the momentum drag of a propulsion system. The same thrust may therefore be produced using less power than an equivalent propulsion system in freestream flow [1]. Research has shown that fuel savings in the region of $5-10 \%$ can be achieved by using boundary layer ingestion [2, 3, 4]. However, significant distortion can negate the efficiency benefits of a BLI system [1].

Performance simulation of the typical podded engines on a conventional transport aircraft is a wellestablished process. The method allows for a relatively simple thrust and drag accounting between the aircraft and its engines. However, this distinction is a greater challenge for a BLI propulsion system. Flow that enters the intake of a BLI propulsion system is not clean free-stream air flow. Rather, it has travelled over the aircraft surface ahead of the intake. The separation of the airframe and propulsion system is therefore more challenging.

Research on the simulation of BLI systems can be broadly split into two categories: computational fluid dynamic (CFD) analyses of the problem as a whole, and lower-order analytical methods. CFD methods are useful for the analysis of the combined aircraft and propulsion system. However, CFD is computationally expensive and is therefore less useful for the preliminary de- sign phase. It is useful to have a rapid, low-order analytical method to evaluate competing technologies or for the process of configuration down-selection.

As an alternative to CFD, analytical methods may be used. Force control volumes have been used to estimate the performance of the propulsion system $[1,5,6]$. These control volumes typically deal with the propulsion system as an isolated or uninstalled system in a similar manner to conventional propulsion system analysis. The boundary layer is generally represented in these methods as a deficit in mass flow, momentum and kinetic energy relative to free-stream flow. The distorted flow of the boundary layer may also result in a reduction in fan or compressor efficiency and a greater total pressure loss in the intake duct. To circumvent the difficulty of thrust-drag bookkeeping, the power balance method developed by Drela assesses the aircraft system as a whole [7]. A similar method was presented by Arntz et al. that uses an exergy analysis of the aircraft and propulsion system [8]. However, such methods are reliant on a more detailed knowledge of the aircraft and propulsion system configuration. In addition, conventional point-mass based aircraft performance models are often reliant on the ability to separate the thrust and drag of the aircraft and propulsion system. Force control volume methods are therefore more suitable for the purposes of preliminary design and performance analyses.

Typically, research on performance of BLI systems focuses on proving the efficiency benefits of a BLI system. Hence, the focus is on sizing and performance at design point. There is therefore a gap in research of tools for off-design simulation of a BLI propulsion system. The identified force control volume methods use a thrust and drag accounting system similar to the uninstalled performance calculations for conventional podded engines [1, 5, 6]. However, BLI systems are inherently integrated and must include aspects of the aircraft configuration to sufficiently represent performance. This work therefore builds on previous force control volumes to attempt to address this particular and key aspect of the technology assessment. The aim is to develop a novel method that includes the integration aspects of the system, whilst remaining flexible enough to accommodate design changes or to be used for different configurations, on a consistent basis. The goal is to create a rapid low-order work flow for use during the preliminary design phase of a BLI propulsion system and aircraft that can be integrated within conventional propulsion system/aircraft performance methods. The method has been applied on a case study aircraft, NASA's N3-X, to demonstrate the developed simulation process. 


\section{Theory}

\subsection{Thrust and Drag Accounting}

The forces produced by an aircraft in flight can be split to belong to either a propulsion system or an airframe force accounting system [9]. Differentiating these forces is a relatively simple matter for an aircraft with podded engines, as there is limited interaction between the engine and airframe. For aircraft with more integrated architectures it becomes more difficult to differentiate between the airframe and the propulsion system [3]. A boundary layer ingesting propulsion system is an integrated system with performance influenced by airflow over the airframe. Performance may therefore be represented by a Net Propulsive Force (NPF), which can include the forces associated with engine integration including the engine cowl and afterbody, spillage drag, and interference drag.

In a BLI system, flow entering the intake has passed over the surface of the aircraft fuselage. It could therefore be argued that the entire fuselage section prior to the intake is a part of the propulsion system control volume. This leads to the requirement to define an appropriate control volume for an integrated BLI propulsion system. Two potential definitions of control volume for a boundary layer ingesting propulsion system are defined here as an internal (propulsion system only) and external (propulsion system and airframe surface up to the leading edge) control volume. In an internal control volume, the airframe drag ahead of the intake is influenced by the suction of the propulsion system. Airframe drag estimates therefore rely on the propulsion system operating point. In an external control volume, the control volume starts at the leading edge, starting in freestream flow and covering the entire region ahead of the intake. However, this control volume includes all the skin friction drag ahead of the propulsion system intake. Propulsion system performance calculations therefore include airframe drag. In both cases, the boundary layer profile is required to estimate the characteristics of flow entering the intake.

Instead, a suitable interface point, $i$, can be chosen which indicates the region where engine thrust and drag can be separated from the aircraft flight conditions, and aircraft drag can be separated from engine performance (Figure 1). This is estimated to lie approximately two inlet heights ahead of the highlight $[10,11]$. In some configurations, the local flow speed just outside the boundary layer may not be the same as the flight velocity. This is generally the case for a BWB configuration, where the aerofoil cross-section means that there is a velocity profile from the leading to trailing edge. It is therefore also useful to define $i 0$ as the local free-stream, i.e. just outside the boundary layer.

A BLI propulsion system is generally mounted on the fuselage surface. In the clean aircraft case, the drag of this wetted surface area is accounted for in the airframe drag calculation. Once the propulsion system is installed, the drag of this surface is now covered by the propulsion system control volume. This portion of the fuselage drag should therefore be removed from the total airframe drag. Hence, the net propulsive force for a BLI system may be defined as follows:

$\mathrm{NPF}=F_{G 9}-F_{G i}-\tau_{w, i} S_{\text {wet }, i}-D_{\text {nacelle }}+\Delta D=D_{\text {aircraft,clean }}$

Where $F_{G 9}$ is the gross thrust, $F_{G i}$ is the momentum drag, $\tau_{w, i} S_{\text {wet }, i}$ is the skin friction of the surface from the interface point to the intake highlight, $D_{\text {nacelle }}$ is the nacelle drag, $\Delta D$ is the skin friction drag of the airframe surface covered by the propulsion system control volume and $D_{\text {aircraft,clean }}$ is the drag of the aircraft without the propulsion system. $F_{G}$ can be defined as follows:

$$
F_{G}=\dot{m} u+A\left(p-p_{0}\right)
$$

The difference between $F_{G 9}$ and $F_{G i}$ is analogous to the conventional net thrust term used in propulsion system performance reporting. For a free-stream propulsor, $F_{G i}$ is equal to $F_{G 0}$. In addition, the skin friction is no longer a part of the control volume. The definition of NPF therefore becomes similar to the conventional thrust definition for a free-stream system. For an aircraft of a fixed size (i.e. $D_{\text {aircraft,clean }}$ constant), the net propulsive force required from the system at design point is constant. The installed BLI system can therefore be simulated independently from the aircraft performance. The only airframe inputs to the propulsion system performance are the local flow conditions.

\subsection{Boundary Layer Flow Characteristics}

Equations which describe the flow in a boundary layer can be derived from the Navier-Stokes equations for viscous flow. These equations reduce to a more easily solvable form than the full Navier-Stokes equations through the application of appropriate limits to the integrals and with the use of velocity profile approximations [12]. The $1 / n^{\text {th }}$ power law relationship provides one form of velocity profile approximation, where a typical value used to approximate a fully developed boundary layer is $n=7$ [13]. In addition to the boundary layer thickness, $\delta$, a number of additional dimensions that define the boundary layer are available: the displacement thickness $\left(\delta^{*}\right)$, momentum thickness $(\theta)$, and 


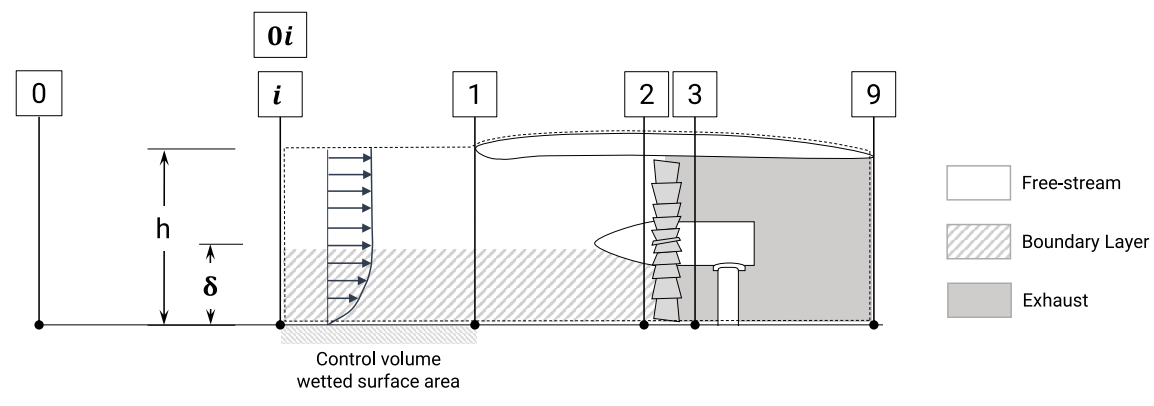

Figure 1: Propulsion system control volume and station definition.

energy thickness $\left(\theta^{*}\right)$ [14]. Each boundary layer thickness term represents the distance by which the surface would have to be displaced in an inviscid flow in order to result in the same mass flow, momentum or kinetic energy as the viscous flow. Boundary layer flow characteristics may be represented as non-dimensional parameters by applying these boundary layer thickness definitions. For the purposes of this research, the mass flow is represented as a non-dimensional parameter, the 'mass flow group' (MAG) [11]:

$$
\mathrm{MAG}=\frac{\dot{m}_{\mathrm{BL}}}{\rho_{0} u_{0 i} \delta w}=\int_{0}^{1} \frac{\rho_{y}}{\rho_{0}} \frac{u_{y}}{u_{0}} \mathrm{~d}(y / \delta)=1-\frac{\delta^{*}}{\delta}
$$

Flow in the boundary layer also has total pressure deficit and a reduction in velocity relative to free-stream flow. These can be calculated as a mass flow-averaged values:

$$
\frac{\bar{P}_{B L}}{P_{0}}=\frac{1}{\dot{m}_{B L}} \int_{B L} \frac{P_{y}}{P_{0}} \mathrm{~d} \dot{m}=\frac{1}{M A G} \int_{0}^{1} \frac{P_{y}}{P_{0}} \frac{u_{y}}{u_{0}} \frac{\rho_{y}}{\rho_{0}} \mathrm{~d}(y / \delta)
$$

$$
\frac{\bar{u}_{B L}}{u_{0}}=\frac{1}{\dot{m}_{B L}} \int_{B L} \frac{u_{y}}{u_{0}} \mathrm{~d} \dot{m}=\frac{1}{M A G} \int_{0}^{1}\left(\frac{u_{y}}{u_{0}}\right)^{2} \frac{\rho_{y}}{\rho_{0}} \mathrm{~d}(y / \delta)
$$

The primary purpose of this step in the process is to obtain three flow characteristics needed for propulsion system performance calculation: mass flow, velocity, and total pressure. Any of the numerous methods available for determining boundary layer characteristics may be applied, provided that they produce the required flow characteristics.

\subsection{Inlet Flow Characteristics for a BLI System}

Depending on its size, flow ingested by the propulsion system may be more than $(h / \delta>1)$, less then $(h / \delta<1)$ or only the boundary layer $(h / \delta=1)$. A higher proportion of ingested free-stream air (high $h / \delta$ ) implies a system with inlet flow characteristics tending to free-stream. In the case where the propulsion streamtube is the same size of the boundary layer, the inlet flow characteristics are represented by Equation 3, Equation 4 and Equation 5. In the case where less than the entire boundary layer is ingested, the flow characteristics for the incoming streamtube may be estimated by changing the limits of the previous integrals to between 0 and the streamtube height, $h$, where $h$ is less than $\delta$. In the final case, ingested flow combines both free-stream and boundary layer flow characteristics. Given the definition of station $i$, the boundary layer flow characteristics and profile can be calculated independently. However, flow characteristics for the streamtube as a whole will be influenced by the ratio of free-stream to boundary layer air, $h / \delta$. For the mass flow, this may be calculated as follows:

$$
\frac{\dot{m}_{\mathrm{total}}}{\rho_{0} u_{0 i} \delta w}=\frac{\dot{m}_{\mathrm{FS}}+\dot{m}_{\mathrm{BL}}}{\rho_{0} u_{0 i} \delta w}=\left(\frac{h}{\delta}-1\right)+\mathrm{MAG}
$$

The total pressure deficit and average velocity of the streamtube flow must be averaged over the entire inlet stream. However, total pressure for flow outside the boundary layer is constant $\left(P_{y}=P_{0}\right)$. This splits the integral into boundary layer ( $y$ between 0 and $\delta$ ) and free-stream flow sections ( $y$ between $\delta$ and $h$ ):

$\frac{\bar{P}_{i}}{P_{0}}=\frac{1}{\dot{m}_{\text {total }}}\left[\int_{0}^{\delta} \frac{P_{y}}{P_{0}} \mathrm{~d} \dot{m}+\int_{\delta}^{h} \mathrm{~d} \dot{m}\right]=\frac{\left(\frac{h}{\delta}-1\right)+\operatorname{MAG}\left(\frac{\bar{P}_{B L}}{P_{0}}\right)}{\left(\frac{h}{\delta}-1\right)+\operatorname{MAG}}$

The average velocity in the combined stream may be likewise calculated by integrating over the entire stream:

$$
\frac{\bar{u}_{i}}{u_{0}}=\frac{\left(\frac{h}{\delta}-1\right)+\operatorname{MAG}\left(\frac{\bar{u}_{B L}}{u_{0}}\right)}{\left(\frac{h}{\delta}-1\right)+\operatorname{MAG}}
$$




\subsection{Off-Design Performance}

A key aspect of developing a new model for simulating a boundary layer ingesting propulsion system was to enable the use of the model over the full aircraft mission profile. The reviewed literature presented at the beginning of the chapter does not provide a method for simulating BLI propulsion systems at off-design. Therefore, this section will detail the extension of the design point method detailed in the previous sections for use at off-design and hence any altitude, Mach number, or propulsion system power setting.

The performance of a propulsion system at off-design can be represented by maps that relate pressure ratio, mass flow, rotational speed and/or efficiency of each propulsion system component. At off-design, the change in mass flow demanded by a propulsor may be represented by the selected component running lines. This depends on the configuration of the components, such as nozzle area, or variable subcomponents such as inlet guide vanes. The mass flow demand may be presented as a non-dimensional mass flow (NDMF) independent from the flight conditions:

$$
\mathrm{NDMF}=\frac{\dot{m} \sqrt{T}}{P}
$$

The running line of the component provides a relationship between the mass flow through the component, its pressure ratio, rotational speed, and efficiency. The mass flow demanded by the operating point of each component must be matched to the operating point of other components within the propulsion system. The size of the inlet stream varies depending on the mass flow demand, with a high mass flow demand resulting in a larger cross-sectional area for the incoming streamtube of air. For a BLI system, the size of the streamtube will have a noticeable effect on the characteristics of the flow entering the intake. An engine operating with a high capture area ratio will ingest predominantly free-stream air, with a very high ratio of $h / \delta$. In contrast, a propulsor operating with a low capture area ratio may ingest predominantly boundary layer. The boundary layer thickness is also a function of the flight velocity, amongst other factors. The flow characteristics required for the non-dimensional mass flow are therefore a function of the size of the capture streamtube and hence the mass flow demand.

Given the definition of station $i$, the boundary layer flow characteristics are determined by the Mach number and altitude, regardless of the propulsion system power setting. These may therefore be calculated independently from the streamtube size. However, given the relationship between flow characteristics and the size of the inlet stream, a mass flow-matching procedure is required to match the upstream mass flow and streamtube size to the mass flow demanded by the propulsor (Figure 2):

1. Use standard mass flow matching procedures to obtain propulsor component operating speed line

2. Determine the boundary layer flow characteristics

3. Guess streamtube height, $h$ to determine streamtube flow characteristics and mass flow

4. Calculate mass flow at the fan face from the mass flow demand, NDMF

5. Repeat from Step 3 until streamtube mass flow matches propulsion system mass flow demand

The mass flow matching method is a generic workflow that is intended to be applicable for any propulsion system configuration. The goal of the mass flow matching process is to determine inlet flow characteristics, given that the capture area ratio and hence $h / \delta$ is initially unknown. The procedure should be included within the matching process for the component operating points to determine the engine's overall operating point. Once the engine's operating point has been determined and the mass flow matching procedure is complete, the performance of the propulsion system may be estimated by following conventional 1D gas dynamics methods.

The method does not assess the behaviour of the boundary layer once it has entered the propulsion system control volume. In addition, the integrals presented in the previous section are 1D. A number of general assumptions are therefore used: Flow at station $i$ is independent of propulsion system mass flow demand. Constant ratio of free stream to boundary layer air, $h / \delta$, from the interface point onwards. Streamtube flow characteristics are averaged from the interface point onwards. Square streamtube cross-section of constant width, $w$. Flow characteristics do not vary significantly along the width of the streamtube.

\subsection{Representing the Efficiency of a BLI System}

The performance benefit of a BLI system is often represented in research in terms of a power saving coefficient (PSC) [2]. This term represents the power consumption of a propulsion system producing a fixed NPF with boundary layer flow in comparison to a free-stream propulsion system. Positive PSC represents a propulsion system that is more efficient than an equivalent in free-stream. However, it is also useful to have a metric to directly identify efficiency of a system, rather than defining a system against which BLI should be compared. A metric analogous to the thrust specific fuel 


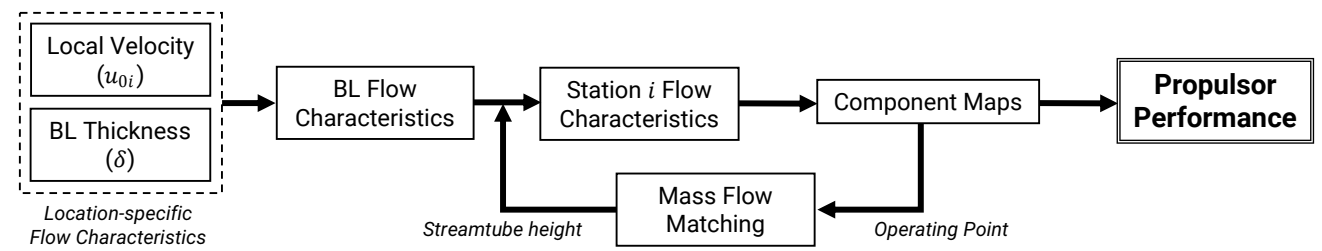

Figure 2: BLI propulsor simulation method at off-design

consumption will also be used here; a 'thrust specific power consumption':

$$
\mathrm{SPC}=\frac{P_{\text {propulsor }}}{\mathrm{NPF}}
$$

This metric represents the power demanded by a propulsor per unit propulsive force. As with specific fuel consumption, a lower SPC implies a more efficient system. This term is useful to help identify a minimum power configuration.

\subsection{Sizing and Design Point Performance}

In the case of a BLI system, changes to the propulsor dimensions (and hence the streamtube size) or location with respect to the airframe will influence the averaged flow characteristics at the interface point. Scaling a propulsor is therefore not a linear relationship between size and propulsive force, unless $h / \delta$ and the propulsor location is fixed. There are two key representations of the influence of propulsor location on local flow characteristics: a variation in local velocity $\left(u_{0 i}=f(x, z)\right)$ and a change in boundary layer thickness $(\delta=f(R e, x))$. Once a propulsor's location is selected, the boundary layer characteristics and propulsor streamtube characteristics may be determined. The inlet flow characteristics for a given value of $h$ can then be used to estimate the performance of the propulsor using conventional one dimensional gas dynamics methods for propulsion system performance. An iterative procedure is required to obtain the propulsor size for the requisite NPF.

The impact of the boundary layer on flow entering the propulsor is represented as three changes relative to free-stream: a total pressure deficit, a reduction in velocity, and a reduction in mass flow. Additional inlet total pressure loss due to the boundary layer and fan/compressor efficiency loss due to distortion may instead be introduced as averaged numerical approximations. For the purposes of this research a NACA-1 forebody and circular arc afterbody was implemented for nacelle drag estimation.

\section{N3-X Case Study Definition}

NASA's N3-X conceptual aircraft [4, 15] (Figure 3) was used as a case study to demonstrate the workflow developed in the research. The N3-X is designed to reduce energy consumption by at least $60 \%$ relative to a conventional 2005 entry-into-service aircraft. In order to achieve this, the aircraft makes use of a number of novel technologies including a blended wing body airframe, turbo-electric distributed propulsion, boundary layer ingestion, and a superconducting electrical transmission system. Publicly available data on the aircraft was applied to demonstrate the analysis possible using the limited information available at an early design stage. This is in keeping with the intended application of the method as a preliminary design tool.

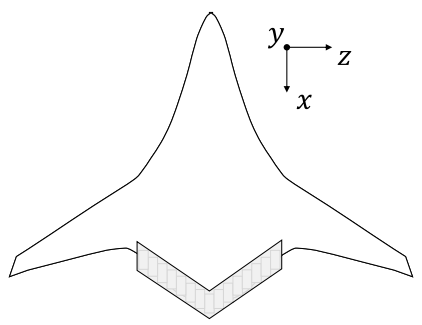

Figure 3: N3-X aircraft top-down sketch (not to scale)

The case study made use of publicly available data on the aircraft and propulsor array configuration [15], and the boundary layer profiles at the centreline of the fuselage [4]. A turbulent flat plate assumption was applied for all flight conditions to estimate boundary layer thickness. To account for the discrepancy between the flat plate assumption and the actual aircraft configuration, the boundary layer thickness was scaled by a constant to match the available boundary layer profiles. Local free-stream Mach number at the edge of the boundary layer from $x / c=0.6$ to $x / c=1.0$ was extracted from the boundary layer profiles, where $c$ is the chord length. In the absence of full CFD data for the airframe, the velocity profile was extended to encompass the entire airframe. This included the assumption that the local free-stream velocity at the edge of the boundary layer 
would be equal at any axial distance $x_{0}$ from the aircraft nose. It was assumed that the airframe velocity profile may be scaled to the flight Mach number.

The array is sized for the aerodynamic design point (ADP) at Mach $0.84,30,000 \mathrm{ft}$ and consists of 15 propulsors with a fan pressure ratio of 1.3 and an adiabatic efficiency of 0.9535 [4]. The net propulsive force required at ADP is $119 \mathrm{kN}$ [16]. The propulsors are assumed to use a variable area floating nozzle to ensure fan stability given the low fan pressure ratios being used for the propulsors [15]. Off-design mass flow matching is therefore required only between the fan and the mass flow of the inlet stream, as the nozzle area is assume to adjust to match the fan operating point. The fans are assumed to operate on a peak efficiency running line. The array is sized such that each propulsor produces the same NPF at design point. As a result, each propulsors has a different mass flow, size, and power demand, mirrored about the centreline. The lowest power fan is located at the centreline, as it ingests the thickest boundary layer and has a relatively lower local velocity. Power demand increases for propulsors at the extreme end of the array, due to a thinner boundary layer and higher local velocity [17]. The total power demand for the propulsor array is $35.2 \mathrm{MW}$ at design point (average of 2.35 MW per propulsor).

\section{Model Validation}

The model was validated against previous simulations for the N3-X propulsion system at design point by Felder et al. [16]. As the research did not include location-related or integration factors, performance was calculated for a propulsor at the centreline of the airframe only. Initially, the predicted inlet stream characteristics were compared to previous results derived from CFD data. Comparison of the inlet stream properties to the NASA results shows an average difference in Mach number of 0.015 and an average $0.2 \%$ difference in boundary layer total pressure deficit [17]. Subsequently, the propulsor size from the sizing procedure was compared. For each case, propulsor net thrust, fan pressure ratio, inlet aspect ratio, and fan efficiency were defined to equal values specified in previous research [16]. The propulsor size predicted by the model was found to match previous predictions to within $4 \%$ [17]. The validation therefore demonstrated that the difference in estimated inlet flow characteristics has only a minor impact on sizing calculations.

\section{Case Study Results}

\subsection{Boundary layer-related Losses}

The 1-D control volume used here does not directly represent losses due to boundary layer distortion. However, the sensitivity of performance to two distortionrelated factors was assessed. The first is a loss in total pressure through the inlet. This term accounts for the turbulent nature of the boundary layer, which may result in reductions in inlet efficiency on top of losses that would normally occur in the intake duct of a free-stream propulsion system. The second loss is a drop in fan efficiency due to the non-uniform velocity and pressure profile at the fan face.

An increase in the total pressure loss through the intake leads to an increase in the propulsor's power consumption (Figure 4a). The power consumption of very low fan pressure ratio propulsors (FPR 1.1-1.2) is strongly influenced by the total pressure deficit in the propulsor, and there is a sharp increase as the deficit increases. In order to achieve the required thrust, the propulsion system requires an increasingly large mass flow to compensate for a reduction in effective fan pressure ratio brought about by the inlet pressure loss. This is obtained by an increase in propulsor size, which increases the ratio $h / \delta$ and brings the inlet average flow characteristics closer to free-stream. A higher fan pressure ratio reduces the mass flow required by the propulsion system and hence decreases the propulsion system size. However, power consumption is increased, as it is a function of both mass flow and enthalpy change. Nonetheless, a higher pressure ratio is beneficial for power consumption in cases of high inlet pressure loss. For a low total pressure loss (e.g. the $0.2 \%$ value quoted in the original research on the N3-X), the propulsor with the lowest power and highest power saving versus a free-stream propulsor is the one with the lowest fan pressure ratio. However, an optimum fan pressure ratio for minimum power begins to emerge as the total pressure loss increases, defining a configuration with the lowest power consumption for the particular duct pressure loss. Decreasing the efficiency of the fan results in an increase in the enthalpy change across the fan. However, there is little change in the thrust produced and hence propulsor size does not need to be increased. This results in only a step increase in the power requirement of the fan as fan efficiency is decreased (Figure 4b). A key conclusion to highlight is that a higher power saving versus a free-stream system may not correspond to the minimum power configuration. A high PSC is therefore not always the lowest power propulsion system option. 


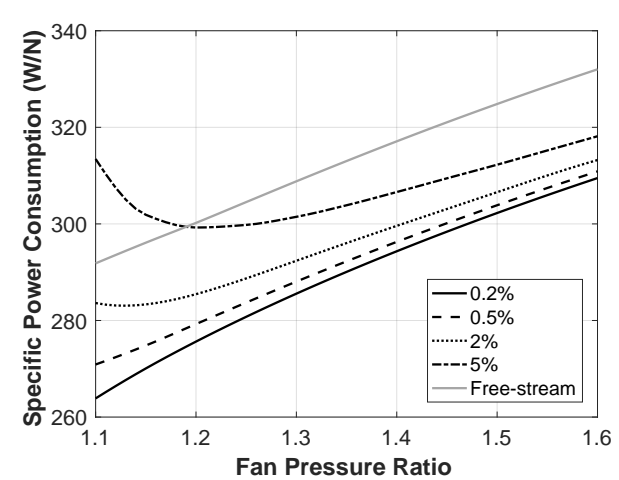

(a) Intake total pressure loss

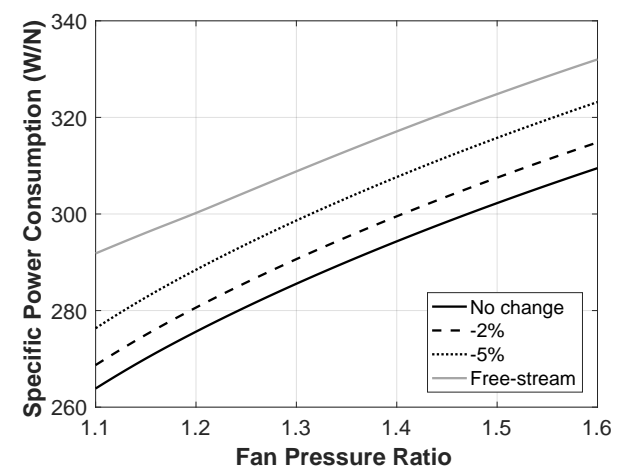

(b) Fan efficiency loss

Figure 4: Influence of efficiency loss on specific power consumption for a propulsor on the N3-X airframe centreline at M0.84 and 30,000 $\mathrm{ft}$ (neglecting nacelle drag).

\subsection{Individual Propulsors}

Each propulsor is sized to produce the same net propulsive force at design point. However, they will perform differently at off-design due to differences in propulsor size and design. In addition, each propulsor in the array is subject to different flow conditions. The performance of two propulsors in the array, the centreline propulsor and the propulsor at the far end of the array, was simulated at the ADP flight conditions, Mach 0.84 at 30,000 ft, (Figure 5) and sea level static (Figure 6) for a range of fan rotational speeds (i.e. power settings).

Both propulsors are sized for the same NPF at design point, therefore, the reduction in net propulsive force as the fan RPM reduces is similar (Figure 5a). However, the centreline propulsor produces slightly more net propulsive force than the end propulsor as the rotational speed is reduced. Both fans are assumed to operate on the same fan map (scaled to their respective nondimensional mass flow). Their running line is therefore similar, with the same relationship between rotational speed, fan pressure ratio, and efficiency. The difference arises due to the different flow characteristics of the two propulsors. Momentum drag for centreline propulsor is lower, due to a thicker boundary layer and slower local flow. The centreline propulsor is therefore able to produce more net thrust than the propulsor at the extreme edge of the array. In addition, the SPC of the centreline propulsor is consistently lower than propulsors further along the span (propulsors from centreline to outer edge plotted on Figure 5b). Reducing the rotational speed of the propulsor reduces its power consumption and increases its propulsive efficiency (due to a lower exhaust velocity), hence SPC improves as the rotational speed is reduced. In addition, $h / \delta$ decreases as rotational speed reduces, due to a lower mass flow demand and hence a lower mass flow ratio. The results also highlight that a minimum SPC point becomes apparent for the propulsors at the edge of the array.

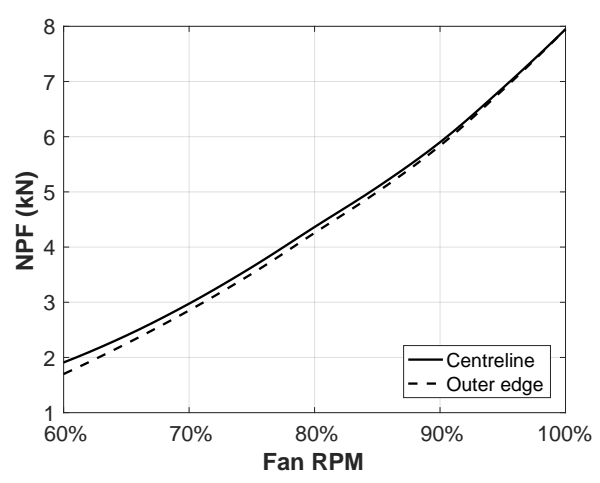

(a) Net propulsive force.

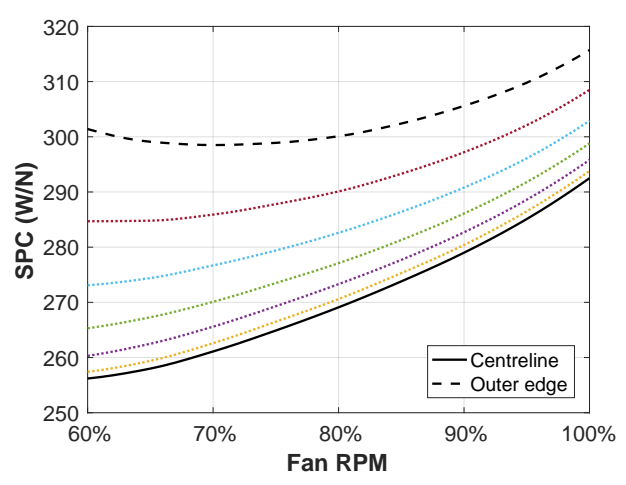

(b) Specific power consumption.

Figure 5: ADP performance of propulsors at the centreline and end of the propulsor array as a function of rotational speed.

At sea level static the airframe boundary layer is negligible, meaning location-specific differences do not 
play a part in performance. In addition, the capture area ratio ratio of sea level static operation means that any ingested boundary layer is negligible in comparison to the ingested free-stream flow $(h / \delta$ tends to infinity). Therefore, the only difference in performance between propulsors is the difference resulting from their size. As the fan efficiencies and inlet flow characteristics are the same, both propulsors have the same specific power consumption for any rotational speed at SLS (Figure 6b). However, the propulsor at the array end is sized for a higher non-dimensional mass flow, as the sizing process assumed that all propulsors must produce the same NPF at design point. The propulsor at the array end therefore produces slightly more thrust than the centreline propulsor at SLS, as there is no difference in momentum drag or inlet flow characteristics as a function of location at static conditions.

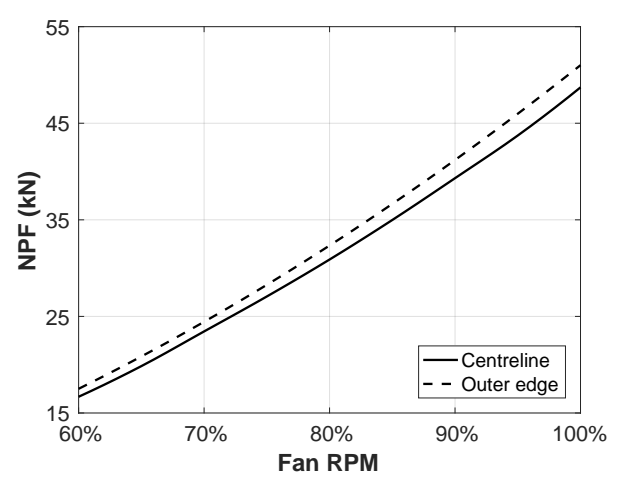

(a) Net propulsive force.

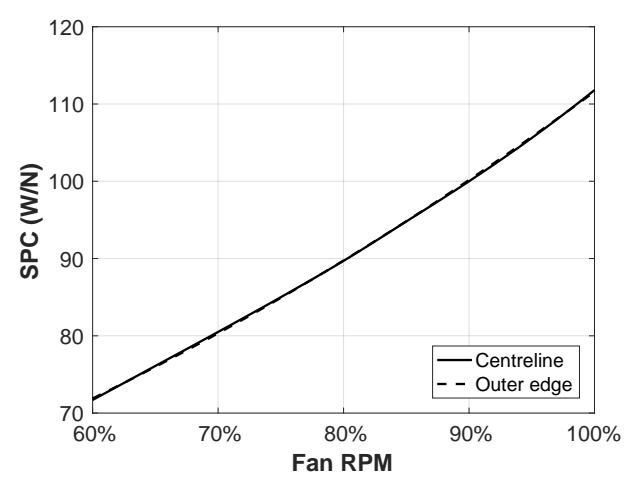

(b) Specific power consumption.

Figure 6: Sea level static performance of propulsors at the centreline and end of the propulsor array as a function of rotational speed.

\subsection{Whole Array}

As with a conventional system, more thrust can be produced at low flight velocity, low altitude operating points (Figure 7b). At low speed, the propulsion system is able to produce increasing amounts of thrust, as the momentum drag reduces. At static conditions, $h / \delta$ tends to infinity, and hence the propulsor effectively operates in free-stream flow. This is matched by a lower specific power consumption at low altitude and low Mach numbers (Figure 6b). Although more thrust can be produced in these conditions, these conditions are also associated with a higher capture area ratio (and hence higher $h / \delta$ ), which slightly reduces efficiency in comparison to a lower $h / \delta$. The overall specific power consumption nevertheless decreases overall for the system, due to the increase in thrust produced. At the aerodynamic design point, the lower efficiency of propulsors at the outer edges of the array reduces the overall efficiency of the array as a whole. In contrast, at low speed, high mass flow ratio (high $h / \delta$ ), the array is able to produce significant thrust as total pressure deficit due to the boundary layer reduces and momentum drag is lower. At these conditions, propulsors at the outer edges are able to produce more thrust for a similar efficiency to the centreline propulsor, as they are sized for a larger non-dimensional mass flow.

\subsection{Conclusions}

This research has presented the development of a work flow to size and simulate the performance of a boundary layer ingesting propulsion system. A control volume has been defined that can be used to separate thrust and drag of an integrated BLI propulsion system. This formulation allows aircraft drag to be presented as a 'clean' aircraft drag term, $D_{\text {aircraft,clean, that is inde- }}$ pendent from the propulsion system configuration. The work flow combines boundary layer theory with conventional 1D gas dynamics methods for the estimation of propulsion system performance. Inlet flow characteristics are estimated as total (mass flow) and mass flowaveraged values (pressure and velocity). With BLI, a propulsor's inlet flow characteristics are a function of streamtube size. A mass flow matching process is therefore required to match the mass flow of the streamtube to the mass flow demand of the propulsion system. The method is presented as a generic tool that may be used to predict the performance of a boundary layer ingesting propulsion system at any flight condition.

The process was demonstrated on a case study aircraft, NASA's blended wing body N3-X with a distributed array of boundary layer ingesting propulsors. Intake total pressure loss was found to have a significant impact on the propulsor's performance for very low pressure ratio fans, whilst fan efficiency loss was found 


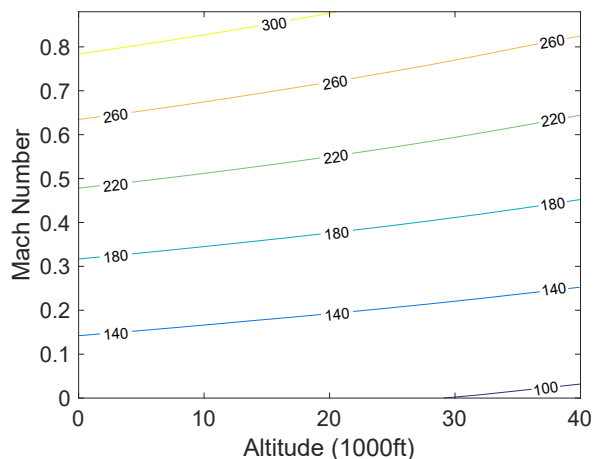

(a) Specific power consumption.

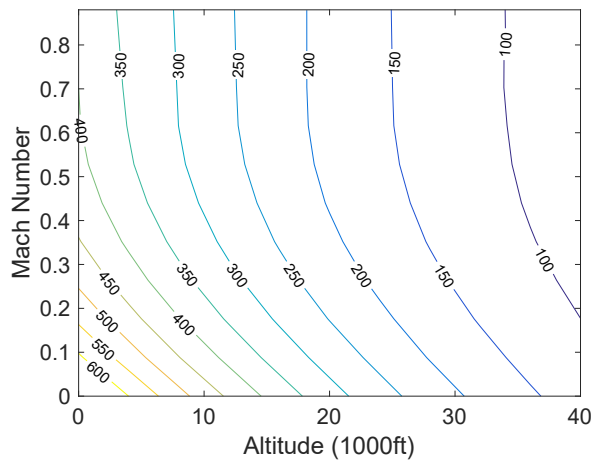

(b) Net propulsive force.

Figure 7: Propulsor array performance as a function of altitude and Mach number at $100 \%$ fan rotational speed

to have a less significant influence. Comparison of individual propulsors identified that location-specific flow characteristics will influence performance. Whilst the propulsors were sized for the same net propulsive force at design point, performance at off-design was found to diverge depending on operating conditions. Efficiency of each propulsor at the aerodynamic design point was found to depend on the power setting, with a minimum power rotational speed emerging for propulsors at the outer edges of the array. In contrast, the efficiency of each propulsors was found to converge at conditions with a high ratio of $h / \delta$, where location-specific difference in flow characteristics become negligible. Finally, the process was used to create a map of the propulsor array's performance at $100 \%$ fan rotational speed as a function of altitude and Mach number. This may then be used in combination with aircraft performance modelling tools to predict the performance of an aircraft using a boundary layer ingesting propulsor array.

\section{Acknowledgements}

The authors would like to extend their gratitude to NASA for making this work possible under grant number NNX13AI78G. Many thanks go also to staff at Cranfield University for their input and to Mr. Darrell Williams for many valuable discussions on developing the performance simulation methods.

\section{References}

[1] A. Plas, M. Sargeant, V. Madani, D. Crichton, E. Greitzer, T. Hynes, C. Hall, Performance of a boundary layer ingesting (BLI) propulsion system, in: 45th AIAA aerospace sciences meeting and exhibit, Reno, NV, USA, 2007, AIAA 2007-450. doi: 10.2514/6.2007-450.

[2] L. H. Smith, Wake ingestion propulsion benefit, Journal of Propulsion and Power 9 (1) (1993) 74-82. doi:10.2514/3. 11487.

[3] L. W. Hardin, G. Tillman, O. P. Sharma, J. Berton, D. J. Arend, Aircraft system study of boundary layer ingesting propulsion, in: 48th AIAA/ASME/SAE/ASEE Joint Propulsion Conference and Exhibit, Atlanta, GA, USA, 2012, AIAA 2012-3993. doi : 10.2514/6.2012-3993.

[4] J. Felder, G. Brown, H. D. Kim, J. Chu, Turboelectric distributed propulsion in a hybrid wing body aircraft, in: 20th International Society for Airbreathing Engines, Gothenburg, Sweden, 2011, ISABE-2011-1340.

[5] J. Felder, H. D. Kim, Control volume analysis of boundary layer ingesting propulsion systems with or without shock wave ahead of the inlet, in: 49th AIAA Aerospace Sciences Meeting, Orlando, FL, USA, 2011, AIAA 2011-222. doi:10.2514/6. 2011-222.

[6] E. A. Valencia, D. Nalianda, P. Laskaridis, R. Singh, Methodology to assess the performance of an aircraft concept with distributed propulsion and boundary layer ingestion using a parametric approach, Proceedings of the Institution of Mechanical Engineers, Part G: Journal of Aerospace Engineering 229 (4) (2015) 682-693. doi : 10.1177/0954410014539291.

[7] M. Drela, Power balance in aerodynamic flows, AIAA journal 47 (7) (2009) 1761-1771. doi : 10.2514/1.42409.

[8] A. Arntz, O. Atinault, A. Merlen, Exergy-based formulation for aircraft aeropropulsive performance assessment: Theoretical development, AIAA Journal 53 (6) (2014) 1627-1639. doi : 10.2514/1. J053467.

[9] NATO Advisory Group for Aerospace Research and Development, Guide to In-Flight Thrust Measurement of Turbojets and Fan Engines, AGARD, 1979, AGARD-AG-237.

[10] A. P. Plas, Performance of a boundary layer ingesting (BLI) propulsion system, Master's thesis (2006).

[11] D. Williams, Application of boundary layer theory to BLI simulation [Personal Communications] (2015).

[12] J. D. Anderson, Fundamentals of Aerodynamics, McGraw-Hill Education, 1991. doi:10.1371/journal.pcbi.1000716.

[13] B. S. Stratford, G. S. Beavers, The calculation of the compressible turbulent boundary layer in an arbitrary pressure gradient a correlation of certain previous methods, Tech. rep., Aeronautical Research Council, ARC-3207 (1961).

[14] H. Schlichting, K. Gersten, Boundary-layer theory, Springer, 2000. doi: 10.1007/978-3-662-52919-5.

[15] J. L. Felder, H. D. Kim, G. V. Brown, Turboelectric distributed propulsion engine cycle analysis for hybrid wing body aircraft, 
in: 47th AIAA Aerospace Sciences Meeting, Orlando, FL, USA, 2009, AIAA 2009-1132. doi : 10.2514/6. 2009-1132.

[16] J. L. Felder, H. D. Kim, G. V. Brown, J. Chu, An examination of the effect of boundary layer ingestion on turboelectric distributed propulsion systems, in: 49th AIAA aerospace sciences meeting including the new horizons forum and aerospace exposition, Orlando, FL, USA, 2011, AIAA 2011-300.

[17] C. Goldberg, D. Nalianda, P. Pilidis, D. MacManus, J. Felder, Installed performance assessment of a boundary layer ingesting distributed propulsion system at design point, in: 52nd AIAA/SAE/ASEE Joint Propulsion Conference, Salt Lake City, UT, USA, 2016, AIAA 2016-4800. doi:10.2514/6. 2016-4800. 
2018-04-23

\section{Method for simulating the performance of a boundary layer ingesting propulsion system at design and off-design}

Goldberg, Chana

Elsevier

Chana Goldberg, Devaiah Nalianda, David MacManus, et al.,Method for simulating the performance of a boundary layer ingesting propulsion system at design and off-design. Aerospace Science and Technology, Volume 78, July 2018, Pages 312-319

https://doi.org/10.1016/j.ast.2018.04.026

Downloaded from Cranfield Library Services E-Repository 